\title{
Tumor necrosis factor-related apoptosis-inducing ligand translates neonatal respiratory infection into chronic lung disease
}

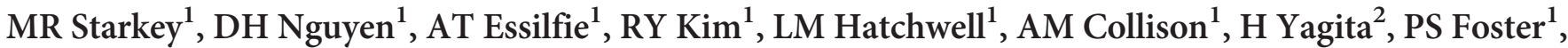 \\ JC Horvat $^{1}, \mathrm{~J}_{\text {Mattes }}{ }^{1,3}$ and PM Hansbro ${ }^{1}$
}

Respiratory infections in early life can lead to chronic respiratory disease. Chlamydia infections are common causes of respiratory disease, particularly pneumonia in neonates, and are linked to permanent reductions in pulmonary function and the induction of asthma. However, the immune responses that protect against early-life infection and the mechanisms that lead to chronic lung disease are incompletely understood. Here we identify novel roles for tumor necrosis factor-related apoptosis-inducing ligand (TRAIL) in promoting Chlamydia respiratory infection-induced pathology in early life, and subsequent chronic lung disease. By infecting TRAIL-deficient neonatal mice and using neutralizing antibodies against this factor and its receptors in wild-type mice, we demonstrate that TRAIL is critical in promoting infection-induced histopathology, inflammation, and mucus hypersecretion, as well as subsequent alveolar enlargement and impaired lung function. This suggests that therapeutic agents that target TRAIL or its receptors may be effective treatments for early-life respiratory infections and associated chronic lung disease.

\section{INTRODUCTION}

Early life respiratory infections with certain bacteria and viruses such as Chlamydia and Mycoplasma and respiratory syncytial virus (RSV) and influenza, respectively, are linked to the development of chronic lung disease. ${ }^{1-4}$ Evidence from clinical and more recently animal model studies link early-life Chlamydia respiratory infection with the development of impaired lung structure and function. ${ }^{3,5-7}$

In humans, Chlamydia pneumoniae and Chlamydia trachomatis are common respiratory pathogens, particularly in early life. Antibodies to C. pneumoniae are found in $50-80 \%$ of adults, and this bacterium is a frequent cause of childhood and community-acquired pneumonia. ${ }^{1}$ C. trachomatis is a common infection of the female reproductive tract and the incidence of vertical transmission to the respiratory tracts of newborns is reported to be as high as $60 \%$, with up to $16 \%$ developing pneumonia. ${ }^{8}$ Chlamydia respiratory infections in early life, particularly those with C. pneumoniae, have been associated with the development of asthma ${ }^{1,9}$ and up to $46 \%$ of pediatric patients with asthma have detectable Chlamydia in their lungs. ${ }^{10,11}$ We have recently demonstrated that neonatal respiratory infection with the natural mouse respiratory pathogen Chlamydia muridarum leads to emphysema-like alveolar enlargement and persistent airway hyperresponsiveness (AHR), ${ }^{3,12}$ which has been confirmed by others. ${ }^{6}$ However, the immunological and inflammatory pathways that are responsible for predisposing to the long-term sequelae of early-life respiratory infection remain unknown.

Tumor necrosis factor-related apoptosis-inducing ligand (TRAIL; also known as tumor necrosis factor ligand, superfamily member 10 (TNFSF10)) is an immune factor that regulates both inflammation and apoptosis. ${ }^{13}$ It was identified based on its sequence homology to Fas ligand and tumor necrosis factor. ${ }^{14}$ TRAIL is expressed by a variety of cells including epithelial cells, monocytes/macrophages, neutrophils, dendritic cells, and T cells. ${ }^{15-20}$ Four cell surface receptors

${ }^{1}$ Priority Research Centre for Asthma and Respiratory Disease, School of Biomedical Sciences and Pharmacy, Faculty of Health, University of Newcastle and Hunter Medical Research Institute, New Lambton Heights, Newcastle, New South Wales, Australia. ${ }^{2}$ Department of Immunology, Juntendo University School of Medicine, Hongo, Bunkyoku, Tokyo, Japan and ${ }^{3}$ Pediatric Respiratory and Sleep Medicine Unit, Newcastle Children's Hospital, Kaleidoscope, New Lambton Heights, Newcastle, New South Wales, Australia. Correspondence: PM Hansbro (Philip.Hansbro@newcastle.edu.au)

Received 3 February 2013; revised 24 July 2013; accepted 13 August 2013; published online 18 September 2013. doi:10.1038/mi.2013.65 
for TRAIL have been identified; death receptor 4 (DR4; also known as TRAIL-R1), DR5 (TRAIL-R2), decoy receptor 1 (DcR1; also know as TRAIL-R3) and DcR2 (TRAIL-R4). ${ }^{21-24}$ Both DR4 and DR5 have functional death domains that signal for apoptosis; however, in mice, DR5 is the only apoptosisinducing TRAIL receptor. DcR1 and DcR2 are expressed in both humans and mice and have no or truncated death domains, respectively, and therefore their activation does not lead to apoptosis. Notably, stimulation of DR4, DR5, and DcR2 can induce activation of NF- $\mathrm{KB}$, which promotes inflammatory and immune responses. ${ }^{21,25}$ TRAIL is important in the immune response to early-life respiratory viral infections including RSV and influenza, which upregulate TRAIL and its receptors, and these viral infections have been linked to persistent pulmonary dysfunction. ${ }^{4,16,18,26-28}$ TRAIL-induced responses are known to promote pulmonary inflammation, mucus hypersecretion, apoptosis of airway epithelial cells, and AHR. ${ }^{15,17,29}$ However, its role in early-life respiratory infection and in driving infection-induced chronic lung disease is unknown.

In the present study, we demonstrate that TRAIL has a previously unrecognized role in promoting neonatal Chlamydia respiratory infection and is pivotal in infection-induced histopathology, inflammation, alveolar enlargement, and persistent AHR. This identifies TRAIL and its receptors as novel therapeutic targets for respiratory Chlamydia infections in early life and for the prevention of infection-induced chronic lung disease.

\section{RESULTS}

The absence of TRAIL results in reduced neonatal Chlamydia respiratory infection-induced histopathology and mucus hypersecretion

To investigate the role of TRAIL during neonatal Chlamydia respiratory infection, wild type (WT) and $\operatorname{Tnfs} f 10^{-/-} \mathrm{BALB} / \mathrm{c}$ mice were infected intranasally (i.n.) with the natural mouse pathogen C. muridarum within $24 \mathrm{~h}$ of birth, and weight gain and Chlamydia load in the lungs were assessed. Infection of WT mice resulted in significantly reduced weight gain from 8 days post infection (d.p.i.) compared with sham-inoculated WT (sham WT) controls (Figure 1a). In contrast, infection of Tnfsf $10^{-1-}$ mice did not result in reduced weight gain compared with shaminoculated $\operatorname{Tnfsf} 10^{-/-}$controls and weight gain was returned to normal (i.e., that of sham-inoculated WT controls). In WT mice, there was an increase in Chlamydia load at 5 d.p.i., infection peaked at 10 d.p.i., and there was a significant decrease at 15 d.p.i. and a further reduction at 20 d.p.i. (Figure 1b). Infection in Tnfs $f 10^{-1-}$ mice did not result in significantly different Chlamydia load to that in WT (C. muridarum WT) controls at 5,10 or 15 d.p.i., although infection was slightly but significantly decreased at 20 d.p.i.

We have previously shown that histopathology (the major determinant of which is pulmonary inflammation) peaks at 15 d.p.i. in neonatal WT mice ${ }^{12}$ and therefore, this is the optimal time point to assess this consequence of infection. Infection of WT mice resulted in significant increases in both histopathology and MSC numbers in the airways at 15 d.p.i. (Figures $\mathbf{1} \mathbf{c}$ and $\mathbf{d}$ ) compared with sham-inoculated WT controls. In contrast, infection of Tnfsf10 $10^{-/-}$mice did not result in marked histopathology or increased MSC numbers compared with sham-inoculated $\operatorname{Tnfs} f \mathrm{fO}^{-/-}$controls. Importantly, there were marked differences in both histopathology and MSC numbers between infected WT mice and infected Tnfsf $10^{-1-}$ mice (Figures $1 \mathbf{e}$ and $\mathbf{f}$ ). There were no major differences between these or any other parameters between sham-inoculated WT and Tnfsf $10^{-1-}$ controls.

\section{Absence of TRAIL results in reduced pulmonary inflammation following neonatal Chlamydia respiratory infection}

Given that infected Tnfsf10 $10^{-/-}$mice had a marked reduction in histopathology at 15 d.p.i., we next examined if there were any differences in the influx of specific types of inflammatory cells into the lung at this time point. Infected WT mice had increased number of alveolar macrophages, inflammatory monocytes, neutrophils, myeloid and plasmacytoid dendritic cells (pDCs), $\mathrm{CD}^{+}{ }^{+}$and $\mathrm{CD} 8{ }^{+} \mathrm{T}$ cells in lung homogenates, compared with sham-inoculated WT controls (Figures 2a-g). Infected Tnfsf $10^{-/-}$also had increased number of alveolar macrophages, inflammatory monocytes, neutrophils, and pDCs, but not mDCs, $\mathrm{CD}^{+}{ }^{-}$or $\mathrm{CD} 8^{+} \mathrm{T}$ cells, compared with sham-inoculated $\mathrm{Tnfsf} 10^{-/-}$controls (Figures 2a-g). Importantly, however, infected Tnfsf $10^{-/-}$mice had significantly reduced number of inflammatory monocytes, $\mathrm{mDCs}, \mathrm{CD} 4^{+}$and $\mathrm{CD} 8{ }^{+} \mathrm{T}$ cells, but not alveolar macrophages, neutrophils, or pDCs, compared with infected WT controls (Figures 2a-g).

Neonatal Chlamydia respiratory infection increases TRAIL production and DR5 expression in the lung

As infection-induced histopathology and inflammation were reduced in $\operatorname{Tnfs} f 10^{-/-}$mice, the effect of infection on TRAIL production and TRAIL receptor expression was assessed. Infection of WT mice resulted in significantly increased TRAIL messenger RNA (mRNA) expression in the lung at 15 d.p.i., but not at 5, 10 or 20 d.p.i., compared with sham-inoculated controls (Figure 3a). There was a concomitant increase in the production of TRAIL protein (Figure $3 \mathbf{b}$ ). Infection increased both the percentage of epithelial cells and alveolar macrophages and the total number of epithelial cells, alveolar macrophages, and inflammatory monocytes expressing TRAIL at 15 d.p.i. (Figures $3 \mathbf{c}$ and d). Infection had no effects on the percentage or total number of endothelial cells, neutrophils, mDCs, pDCs, $\mathrm{CD}^{+}$or $\mathrm{CD}^{+}{ }^{+} \mathrm{T}$ cells expressing TRAIL. Neutrophils, $\mathrm{mDCs}, \mathrm{pDCs}, \mathrm{CD}^{+}{ }^{+}$and $\mathrm{CD} 8^{+} \mathrm{T}$ cells expressed negligible amounts of TRAIL in our model. Infection increased DR5 mRNA expression in the lung at 15 d.p.i., but not 5,10 , or 20 d.p.i. compared with sham-inoculated controls (Figure 3e). No change in DcR1 or DcR2 mRNA expression was observed at any time point (Figures $\mathbf{3} \mathbf{f}$ and $\mathbf{g}$ ).

\section{Neonatal Chlamydia respiratory infection-induced DR5 expression and apoptosis, which is associated with alveolar enlargement}

We have previously shown that infection of neonatal WT $\mathrm{BALB} / \mathrm{c}$ mice results in alveolar enlargement that persists into 

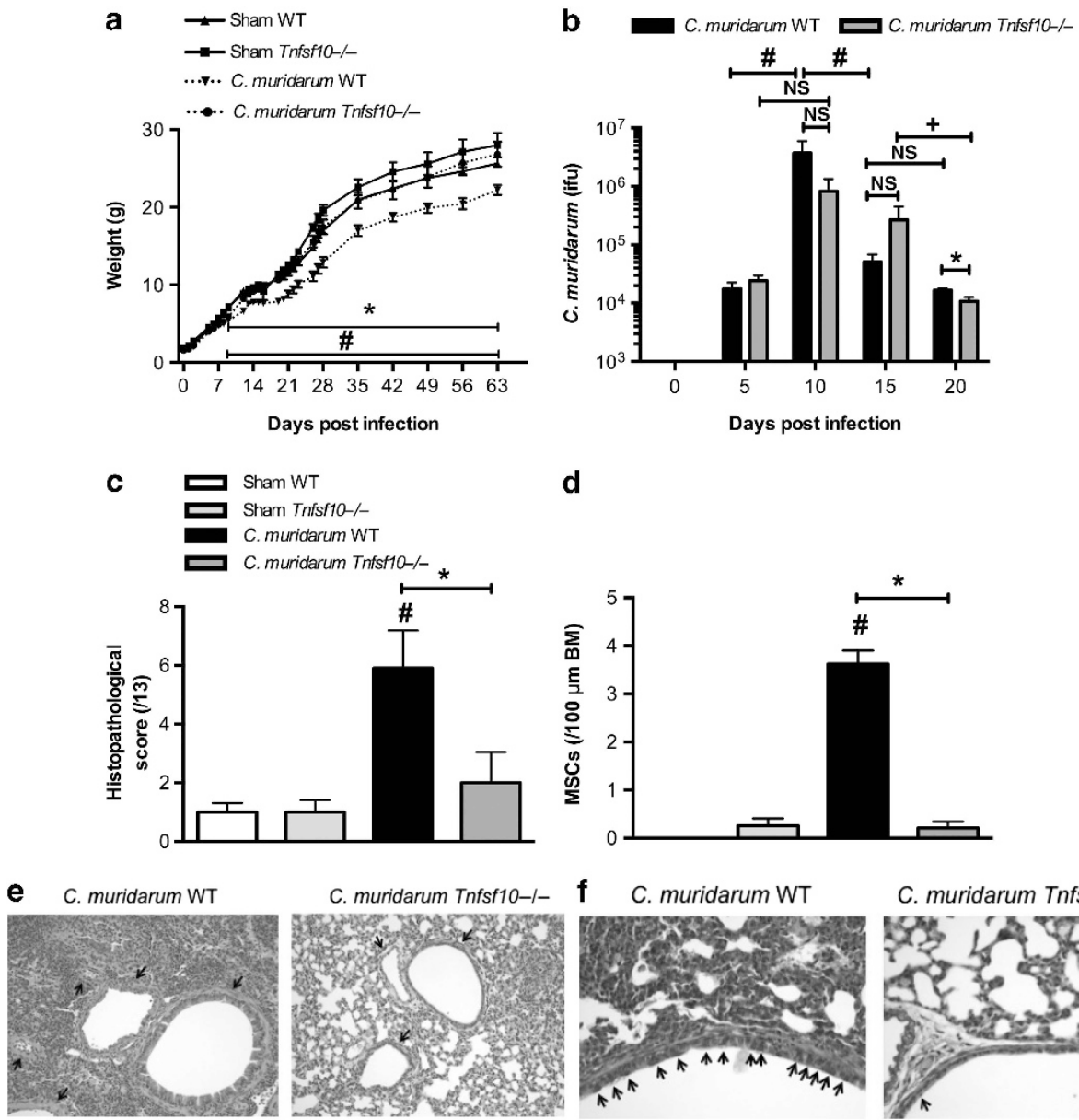

d
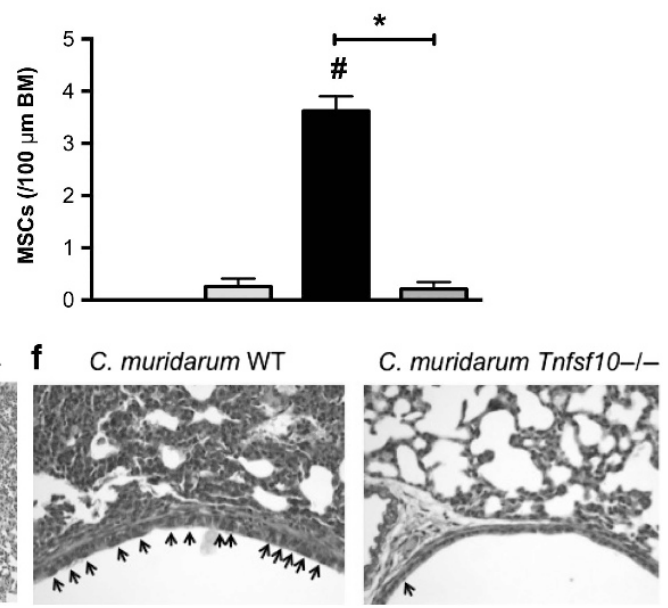

Figure 1 The absence of tumor necrosis factor-related apoptosis-inducing ligand (TRAIL) results in reduced neonatal Chlamydia respiratory infectioninduced histopathology and mucus hypersecretion. Wild type (WT) or Tnfsf1 $0^{-1}$ mice were infected with 400 ifu of $C$. muridarum or sham-inoculated within $24 \mathrm{~h}$ of birth. (a) Weight gain from birth. (b) Chlamydia load in lung homogenates. (c) Histopathology 15 d.p.i. (d) Mucus-secreting cells (MSCs) per $100 \mu \mathrm{m}$ basement membrane (BM) 15 d.p.i. Representative images of (e) hematoxylin and eosin (H\&E) and (f) periodic acid-Schiff (PAS)-stained lung sections from infected WT (left) and infected Tnfsf $10^{-/-}$mice (right) at 15 d.p.i. Results are represented as mean \pm s.e.m. ( $\left.n \geqslant 6\right)$, from 2-3 independent experiments with 3-4 mice in each. " represents $P<0.05$ compared with sham-inoculated WT controls (a,c,d) or between time points in infected WT (b). * represents $P<0.05$ compared with infected WT. ${ }^{+}$represents $P<0.05$ compared between time points in infected Tnfsf10 ${ }^{-/-}$. NS, not significantly different. A full color version of this figure is available at the Mucosal Immunology Journal online.

adulthood, ${ }^{3}$ and this has been recently supported by the studies of others. ${ }^{6}$ Here we confirm this observation and show that the infection of neonatal WT mice results in significantly enlarged alveoli at 61 d.p.i., compared with sham-inoculated WT controls (Figure 4a). By contrast, infection of neonatal $\operatorname{Tnfs} f 10^{-/-}$mice had no effect on alveolar size, compared with sham-inoculated Tnfsf $10^{-/-}$controls. Thus, significantly, infected Tnfsf $10^{-/-}$ mice are protected against infection-induced alveolar enlargement, with alveolar diameter returned to baseline (i.e., to that of sham-inoculated WT controls).

We next sought to determine if differences in DR5 expression and apoptosis were associated with alveolar enlargement in infected WT and Tnfsf $10^{-1-}$ mice. In WT mice, TRAIL and DR5 mRNA were upregulated at 15 d.p.i. (Figures 3a and b), so we assessed DR5 expression and apoptosis of lung epithelial and endothelial cells at this time point. Infection of both neonatal WT and $\operatorname{Tnfs} f 10^{-/-}$mice resulted in increased DR5-positive epithelial $\left(\mathrm{T} 1 \alpha^{+}\right)$and endothelial $\left(\mathrm{CD} 1^{+}\right)$cells in the lung at 15 d.p.i. (Figure $4 \mathbf{b}$ ).
This was associated with a significant increase in epithelial and endothelial cell apoptosis in infected WT mice (Figure 4c). However, infection of neonatal $\mathrm{Tnfs} f \mathrm{fO}^{-/-}$mice did not induce apoptosis of lung epithelial or endothelial cells, compared with sham-inoculated (sham Tnfsf $10^{-/-}$) controls.

We next investigated whether infection was required for alveolar enlargement by administering recombinant (r) TRAIL or agonistic anti-DR5 antibody to the lungs of naïve neonatal WT mice (day 0-18) in the absence of infection. Stimulation of these pathways during early life in the absence of infection had no effect on alveolar size in later life (day 61), compared with isotype-treated controls (Figure $\mathbf{4 d}$ ).

\section{Neonatal Chlamydia respiratory infection promotes persistent AHR in a TRAIL-dependent manner}

We have previously shown that neonatal Chlamydia respiratory infection results in persistent AHR in mice characterized by increased transpulmonary resistance and decreased dynamic compliance in response to methacholine. ${ }^{3,12}$ Here 

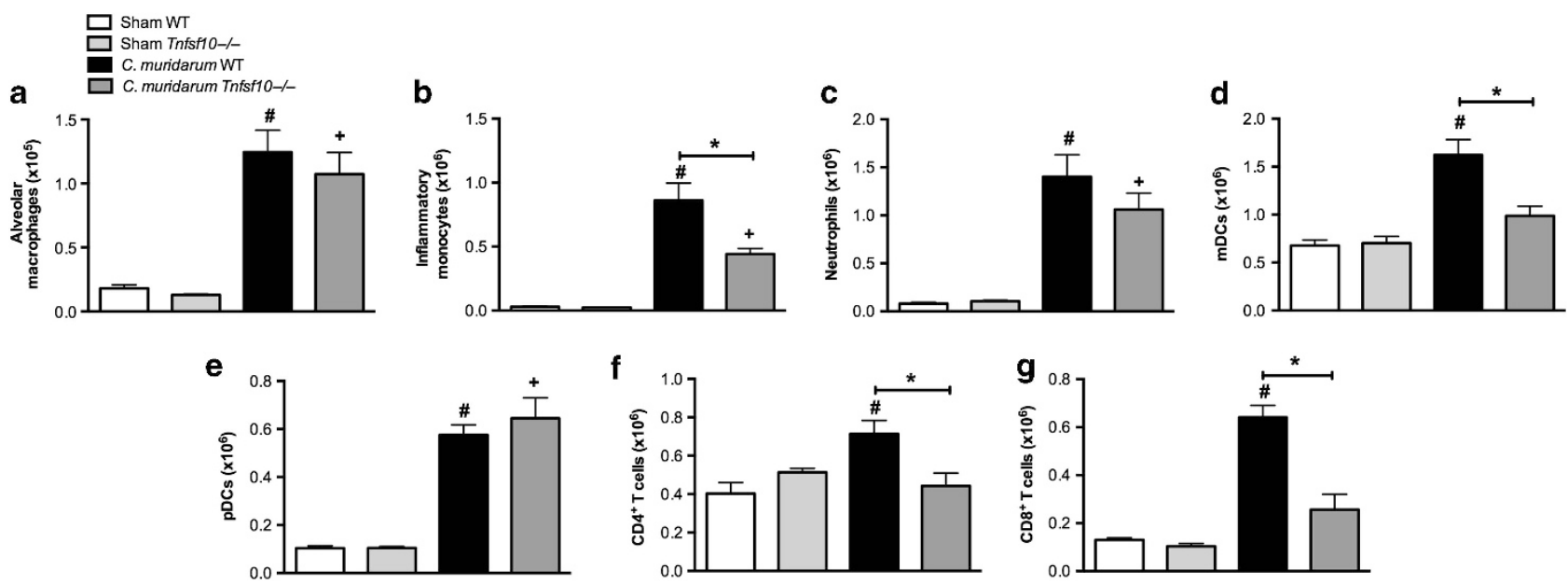

Figure 2 Absence of tumor necrosis factor-related apoptosis-inducing ligand (TRAIL) results in reduced pulmonary inflammation following neonatal Chlamydia respiratory infection. Wild-type (WT) or Tnfsf $10^{-1-}$ mice were infected with 400 ifu of $C$. muridarum or sham-inoculated within $24 \mathrm{~h}$ of birth and pulmonary inflammation assessed at 15 d.p.i. by flow cytometry. (a) Alveolar macrophages. (b) Inflammatory monocytes. (c) Neutrophils. (d) mDCs. (e) pDCs. (f) CD4 ${ }^{+} \mathrm{T}$ cells. (g) CD8 ${ }^{+} \mathrm{T}$ cells. Results are represented as mean \pm s.e.m. $(n \geqslant 6)$, from 2-3 independent experiments with $3-4$ mice in each. \# represents $P<0.05$ compared with sham-inoculated WT controls. ${ }^{+}$represents $P<0.05$ compared with sham-inoculated Tnfsf10 ${ }^{-1-}$ controls.

*represents $P<0.05$ compared with infected WT.

we confirm this observation and show that infection of neonatal WT mice significantly increased AHR, compared with shaminoculated WT controls (Figures 5a and $\mathbf{b}$ ). By contrast, and importantly, infected Tnfsf $10^{-/-}$mice did not develop AHR, as measurements of transpulmonary resistance and dynamic compliance in response to methacholine were not significantly different from sham-inoculated Tnfsf $10^{-/-}$controls and were returned to baseline (i.e., to that of sham-inoculated WT controls).

To determine whether it was the infection or increases in TRAIL or DR5 that was required for induction of persistent AHR, rTRAIL, or agonistic anti-DR5 antibody was administered into the lung of neonatal WT mice in the absence of infection (day 0-18). Importantly, exogenous TRAIL or stimulation of DR5 during early life, in the absence of infection, had no significant effect on AHR in later life (day 61) compared with isotype-treated controls (Figures $\mathbf{5 c}$ and $\mathbf{d}$, and Supplementary Figures S1a-h online).

\section{Blockade of DcR2 during neonatal Chlamydia respiratory infection reduces histopathology, NF-кB activation, and AHR}

We then assessed the role of the TRAIL decoy receptors DcR1 and DcR2 in Chlamydia respiratory infection and infectioninduced pathology. Infected WT mice were treated with blocking antibodies against DcR1 or DcR2 throughout the course of infection. Blockade of DcR1 or DcR2 had no effect on Chlamydia load, MSC numbers around the airways, or the activity of RelB, p65, and p50 at 15 d.p.i., or alveolar size and dynamic compliance at 61 d.p.i. compared with infected isotype-treated (C. muridarum + isotype) controls (Figures 6a,c-f,h). However, blockade of DcR2, but not DcR1, significantly decreased histopathology (pulmonary inflammation (Figure 6b)) and NF- $\mathrm{BB}$ p52 activity (Figure 6d) in the lungs at 15 d.p.i. compared with isotype-treated (C. muridarum + Isotype) controls. Importantly, blockade of DcR2, but not DcR1, during infection also decreased transpulmonary resistance at 61 d.p.i. (Figure 6g).

\section{Neutralization of TRAIL during neonatal Chlamydia respiratory infection reduces histopathology, mucus} hypersecretion, alveolar enlargement, and persistent AHR Clinical signs (i.e., reduced weight gain) of neonatal Chlamydia respiratory infection in mice developed by 8 d.p.i. (Figure 1). Therefore, in order to assess the potential of targeting TRAIL therapeutically, WT neonatal mice were treated with neutralizing anti-TRAIL antibody from 8-18d.p.i., and the effect on Chlamydia load, histopathology, mucus hypersecretion, lung structure, and AHR were assessed. Neutralization of TRAIL did not have a significant effect on Chlamydia load at 10 or 15 d.p.i. (Figure 7a), but significantly reduced histopathology and MSC numbers around the airways at 15 d.p.i. compared with infected isotype-treated controls (Figures $\mathbf{7 b}$ and $\mathbf{c}$ ). Importantly, neutralization of TRAIL also effectively reduced the consequences of early-life infection in adulthood, preventing infection-induced alveolar enlargement, and persistent AHR (decreased transpulmonary resistance and increasing dynamic compliance in response to methacholine) at 61 d.p.i. (Figures $7 \mathbf{d}$ and $\mathbf{f}$ ).

\section{DISCUSSION}

In this study, we discovered a previously unrecognized role for TRAIL in the immune response to early-life Chlamydia respiratory infection that predisposes to acute and chronic pathology. Using a combination of TRAIL-deficient mice and neutralizing antibodies that target TRAIL or its receptors, we demonstrate that TRAIL promotes clinical signs (weight loss), and infection-induced histopathology, inflammation, mucus 


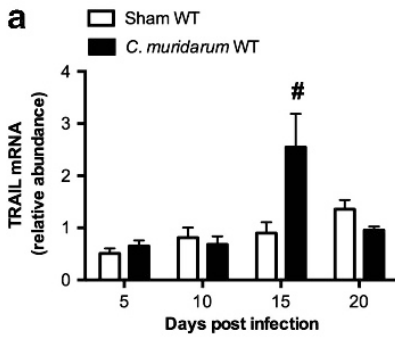

b
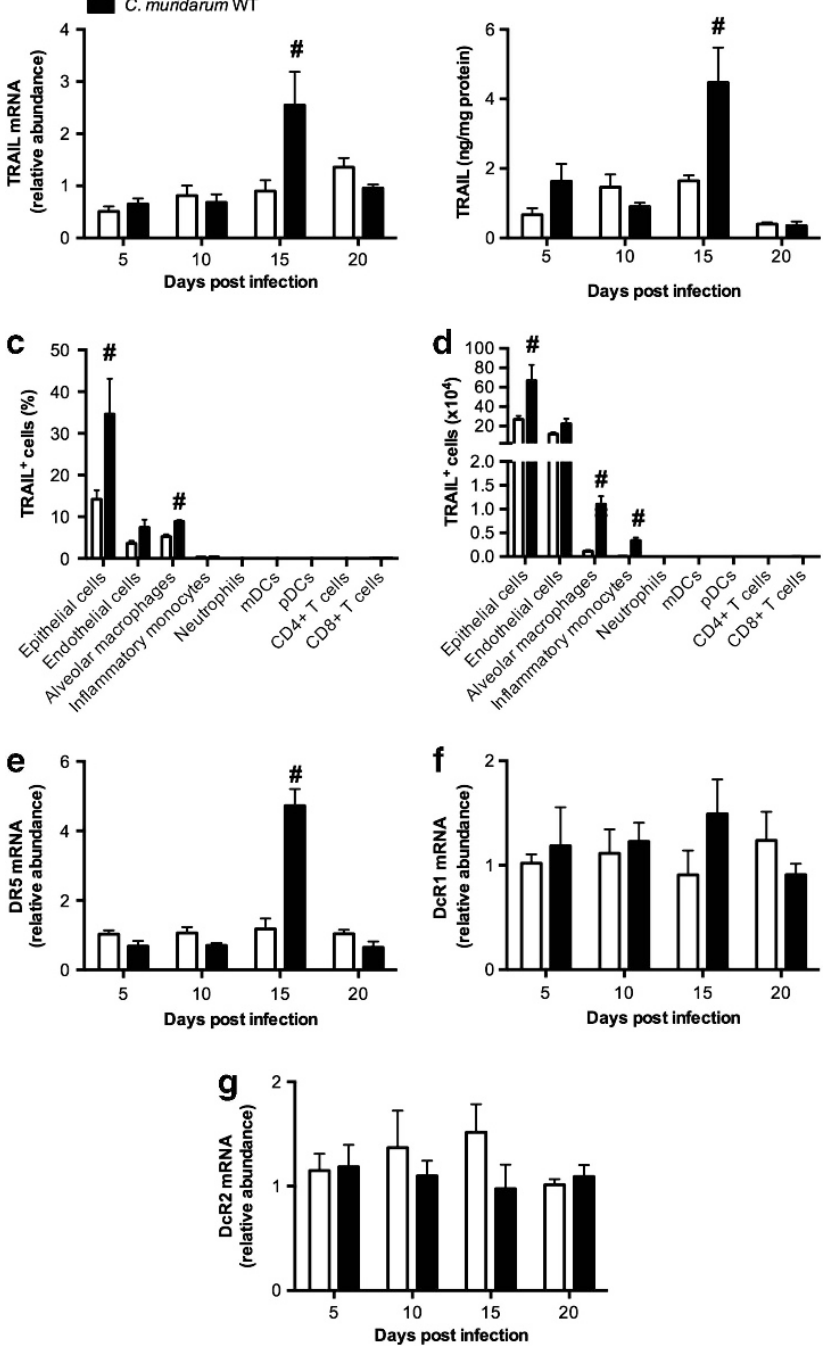

Figure 3 Neonatal Chlamydia respiratory infection increases tumor necrosis factor-related apoptosis-inducing ligand (TRAIL) production and DR5 expression in the lung. Wild-type (WT) mice were infected with 400 ifu of $C$. muridarum or sham-inoculated within $24 \mathrm{~h}$ of birth. (a) TRAIL messenger RNA (mRNA) expression and (b) TRAIL protein levels in whole-lung homogenates. (c) Percentage and (d) total number of epithelial, endothelial, alveolar macrophages, inflammatory monocytes, neutrophils, myeloid dendritic cells, plasmacytoid DCs (pDCs), CD4 ${ }^{+}$T cells and CD8 ${ }^{+}$T cells in whole-lung homogenates at 15 d.p.i. (e) DR5, (f) DcR1 and (g) DcR2 mRNA expression in lung homogenates. Results are represented as mean \pm s.e.m. $(n \geqslant 6)$, from $2-3$ independent experiments with 3-4 mice in each. " represents $P<0.05$ compared with shaminoculated WT controls.

hypersecretion, alveolar enlargement, and AHR. Most importantly, therapeutic targeting of TRAIL during infection decreases these sequelae of infection. Our study further highlights the emerging role of TRAIL in the immune response to respiratory infections.

In our study, we used C. muridarum, as this is a natural mouse pathogen and it requires low-level inocula to induce a productive infection, which replicates the infectious, immunological, and pathological progression of disease in humans, as we have previously published. ${ }^{1,12}$ It is therefore the most appropriate strain to use to study host-pathogen relationships

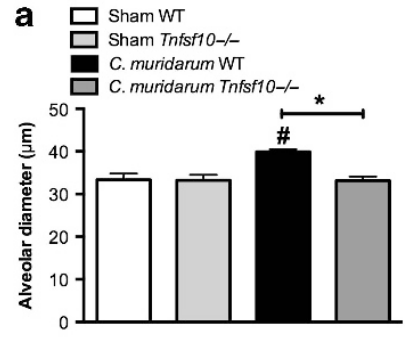

b
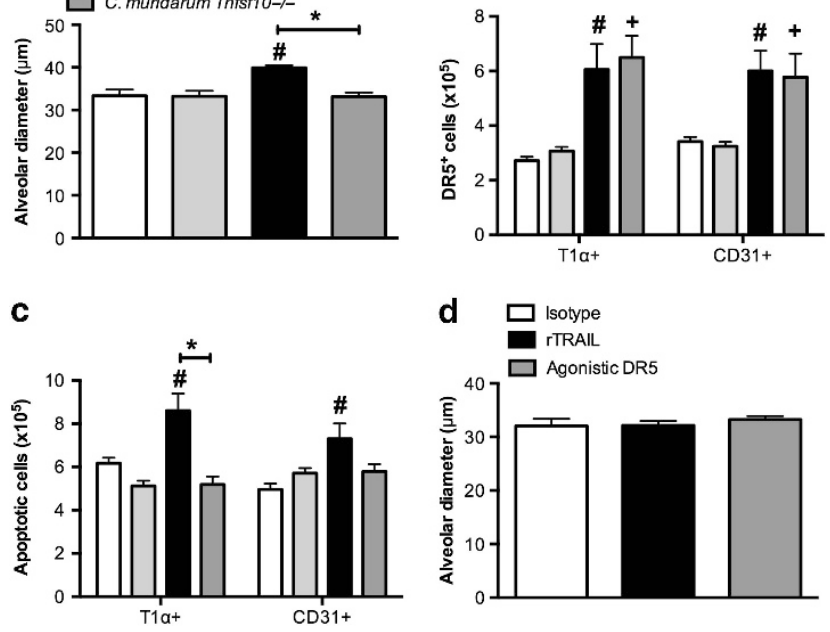

Figure 4 Neonatal Chlamydia respiratory infection induces DR5 expression and apoptosis, which is associated with alveolar enlargement. Wild-type (WT) or Tnfsf10 $10^{-1-}$ mice were infected with 400 ifu of C. muridarum or sham-inoculated within $24 \mathrm{~h}$ of birth $(\mathbf{a}-\mathbf{c})$. In separate experiments, naïve WT mice were administered rTRAIL, agonistic antiDR5 antibody, or isotype control intranasally every 3 days from 0-18 d.p.i. (d). (a) Alveolar diameter at 61 d.p.i. (b) Alveolar epithelial and endothelial cells positive for DR5 at 15 d.p.i. (c) Alveolar epithelial and endothelial cell apoptosis at 15 d.p.i. (d) Alveolar diameter at 61 d.p.i. Results are represented as mean \pm s.e.m. $(n \geqslant 6)$, from $2-3$ independent experiments with 3-4 mice in each. " represents $P<0.05$ compared with shaminoculated WT controls. + represents $P<0.05$ compared with shaminoculated Tnfsf $10^{-1-}$ controls. *represents $P<0.05$ compared with infected WT.
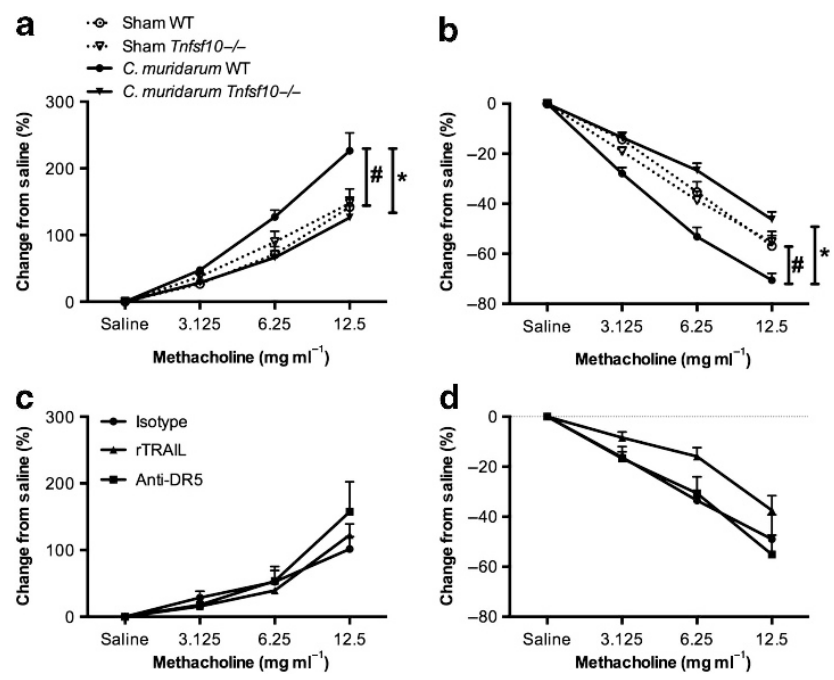

Figure 5 Neonatal Chlamydia respiratory infection promotes persistent airway hyperresponsiveness (AHR) in a tumor necrosis factor-related apoptosis-inducing ligand (TRAIL)-dependent manner. Wild-type (WT) or Tnfsf $10^{-1-}$ mice were infected with 400 ifu C. muridarum or shaminoculated within $24 \mathrm{~h}$ of birth $(\mathbf{a}, \mathbf{b})$. In separate experiments, naïve WT mice were administered rTRAIL, agonistic anti-DR5 antibody or isotype control intranasally every 3 days from 0 to 18 d.p.i. (c,d). AHR was assessed at 61 d.p.i. (a-c) Transpulmonary resistance and (b-d) dynamic compliance represented as percentage change from baseline (saline) in response to inhaled methacholine. Results are represented as mean \pm s.e.m. $(n \geqslant 6)$, from $2-3$ independent experiments with $3-4$ mice in each. " represents $P<0.05$ compared with sham-inoculated WT controls. *represents $P<0.05$ compared with infected WT. 


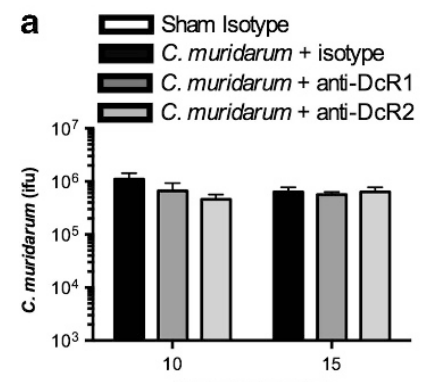

b
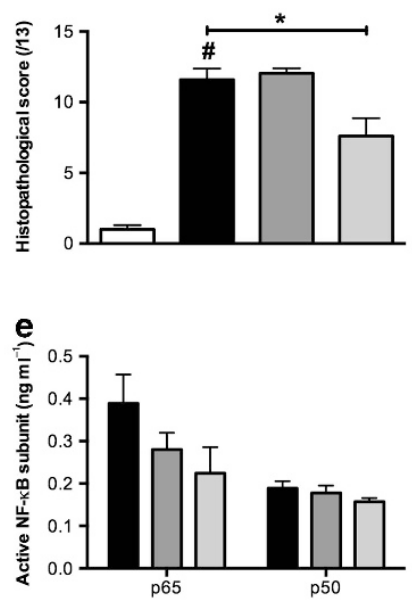

C
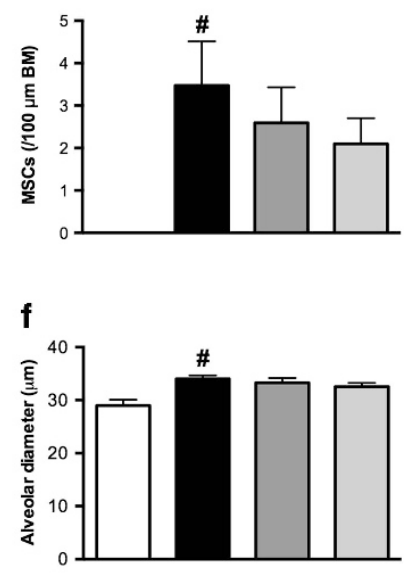
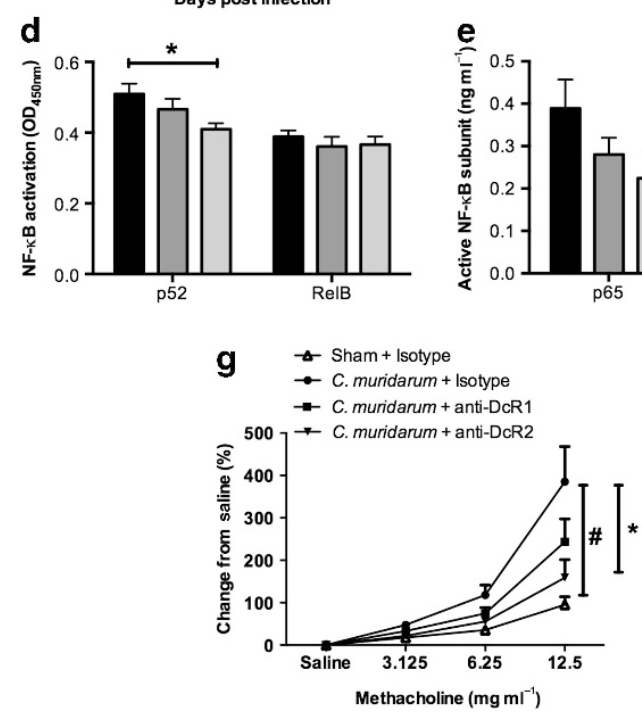

h

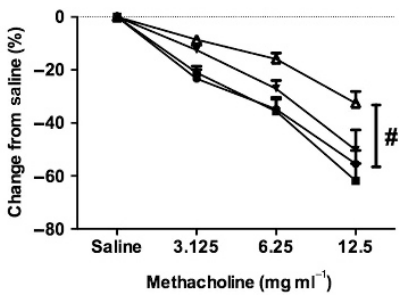

Figure 6 Blockade of DcR2 during neonatal Chlamydia respiratory infection reduces histopathology, NF- $\mathrm{B}$ activity, and airway hyperresponsiveness (AHR). Wild-type (WT) mice were infected with 400 ifu C. muridarum or sham-inoculated within $24 \mathrm{~h}$ of birth and administered neutralizing anti-DcR1 or anti-DcR2 antibody or isotype control intranasally every 3 days from day 0 to 18 . (a) Chlamydia load at 10 and 15 d.p.i. (b) Histopathology at 15 d.p.i. (c) Mucus-secreting cells (MSCs) per $100 \mu \mathrm{m}$ basement membrane (BM) at 15 d.p.i. (d) NF- $\kappa B$ p52 and RelB activity in lung homogenates at 15 d.p.i. (e) NF-kB p65 and p50 activity in lung homogenates at 15 d.p.i. (f) Alveolar diameter at 61 d.p.i. (g) Transpulmonary resistance and (h) dynamic compliance represented as percentage change from baseline (saline) in response to inhaled methacholine at $61 \mathrm{~d}$.p.i. Results are represented as mean \pm s.e.m. $(n \geqslant 6)$, from $2-3$ independent experiments with 3-4 mice in each. ${ }^{*}$ represents $P<0.05$ compared with sham-inoculated isotype-treated controls. *represents $P<0.05$ compared with infected isotype-treated controls.

in mice. In contrast, the human pathogen C. pneumoniae requires a large challenge dose to induce an immune response and does not form a productive infection in mice. Thus, these are clearances rather than infection models and do not replicate what is observed in humans.

Infected $\operatorname{Tnfsf} 10^{-1-}$ mice had normal weight gain during neonatal Chlamydia respiratory infection, which correlated with reduced histopathology, mucus hypersecretion, and infiltration of inflammatory monocytes, mDCs, and $\mathrm{CD} 44^{+}$ and $\mathrm{CD} 8{ }^{+} \mathrm{T}$ cells into the lung. This is in contrast to infected WT mice, which displayed reduced weight gain, and increased histopathology, mucus hypersecretion, and infiltration of all major inflammatory leukocyte subsets examined into the lung. These observations expand on our previous studies, which show that TRAIL drives inflammation and mucus hypersecretion in non-infectious settings. ${ }^{15}$ Intranasal administration of rTRAIL to naïve adult $\mathrm{BALB} / \mathrm{c}$ mice increased the number of mDCs and $\mathrm{CD}^{+}{ }^{+} \mathrm{T}$ cells in the lung and promoted MSC hypersecretion. ${ }^{15}$ Furthermore, the use of Tnfsf $10^{-1-}$ mice and silencing of TRAIL using specific small interfering RNA (siRNA) decreased pulmonary inflammation and MSC numbers during ovalbumin-induced allergic airway disease (AAD) ${ }^{15}$

Surprisingly, the absence of TRAIL had minimal effects on Chlamydia load, with a small but significant decrease in the bacterial burden observed only at 20 d.p.i.. Thus, we propose that the major impact of TRAIL is on immune responses to Chlamydia and in the sequelae of infection rather than on the infection itself. Our observations are in contrast to respiratory pneumococcal infection in adult C57BL/6 mice, where TRAIL deficiency lead to more severe infection and increased mortality. ${ }^{30}$ This suggests that TRAIL may have a protective role in pneumococcal pneumonia.

Interestingly, following neonatal Chlamydia respiratory infection, there was a concomitant increase in TRAIL mRNA expression and protein levels in the lung that correlated with the peak of inflammation. We identified $\mathrm{T} 1 \alpha^{+}$epithelial cells and macrophages/monocytes as the major sources of cell surfacebound TRAIL in the lung. We have previously shown that the airway epithelium was a dominant source of TRAIL during $\mathrm{AAD},{ }^{15}$ and others have shown that macrophages expressing TRAIL are important in influenza-induced alveolar leakage and 

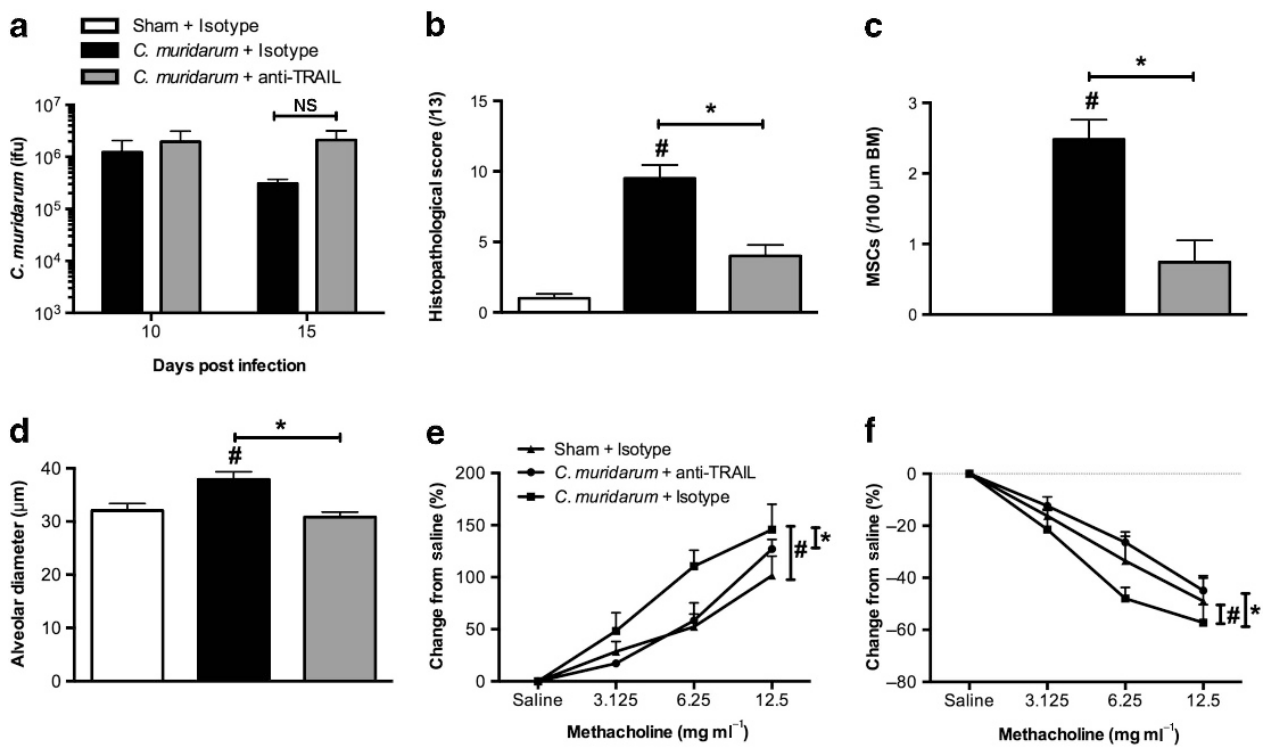

Figure 7 Neutralization of tumor necrosis factor-related apoptosis-inducing ligand (TRAIL) during neonatal Chlamydia respiratory infection reduces infection-induced histopathology, mucus hypersecretion, alveolar enlargement, and persistent airway hyperresponsiveness (AHR). Wild-type (WT) mice were infected with 400 ifu C. muridarum or sham-inoculated within $24 \mathrm{~h}$ of birth and administered neutralizing anti-TRAIL antibody or isotype control intraperitoneally every 2 days from 8 to 18 d.p.i. (a) Chlamydia load at 10 and 15 d.p.i. (b) Histopathology at 15 d.p.i. (c) Mucus-secreting cells (MSCs) per $100 \mu \mathrm{m}$ basement membrane (BM) at 15 d.p.i. (d) Alveolar diameter at 61 d.p.i. (e) Transpulmonary resistance and (f) dynamic compliance represented as percentage change from baseline (saline) in response to inhaled methacholine at 61 d.p.i. Results are represented as mean \pm s.e.m. ( $n \geqslant 6)$, from $2-3$

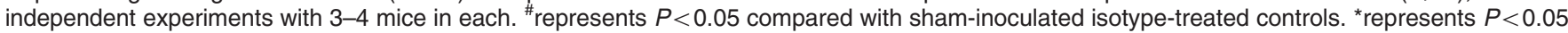
compared with infected isotype-treated controls. NS, not significantly different.

related mortality. ${ }^{17}$ Chlamydia can infect both epithelial cells and macrophages, ${ }^{31,32}$ and infection of these cells may increase the expression TRAIL. Additionally, Chlamydia-induced type I interferons may induce the expression of TRAIL in the lung. ${ }^{33}$ Furthermore, infection-induced infiltration of inflammatory monocytes expressing TRAIL may also contribute to TRAILdependent pathology. While TRAIL expression on the cell surface of neutrophils in our model was negligible, these cells have been shown to be a major source of secreted TRAIL in a mouse model of pneumococcal pneumonia. ${ }^{30}$ Thus, it is possible that neutrophils may also contribute to the increased levels of TRAIL in the lung following neonatal Chlamydia respiratory infection. Others have also shown that $\mathrm{T}$ cells express TRAIL on their cell surface following viral infection. ${ }^{16}$ However, Chlamydia, unlike respiratory viruses, induces negligible TRAIL expression on $\mathrm{CD} 4^{+}$and $\mathrm{CD}^{+}{ }^{+} \mathrm{T}$ cells. Another explanation is that neonatal (as opposed to adult) $\mathrm{T}$ cells may not increase their expression of TRAIL in response to infection. This may help to limit apoptosis following T-cell driven immune responses, which may be important in the growth of the developing lung.

We have previously shown that neonatal, but not infant or adult, Chlamydia respiratory infection leads to emphysemalike alveolar enlargement that persists into adulthood, ${ }^{3}$ which has recently been confirmed by others. ${ }^{6}$ In this study, we demonstrate the mechanism involved. We show that the infection induces the upregulation of DR5 on $\mathrm{T} 1 \alpha^{+}$lung epithelial and $\mathrm{CD} 31^{+}$cells, which were probably endothelial cells, and others have shown that increases in this receptor sensitizes these structural cells to TRAIL-induced apoptosis. ${ }^{27}$
CD31 is the most commonly used endothelial cell marker, although other cells such as macrophages and neutrophils can also express CD31. Tnfs $f 10^{-/-}$mice had increased DR5 expression on epithelial and endothelial cells, but in the absence of TRAIL, the number of epithelial cells that were apoptotic was reduced back to baseline. These data indicate that TRAIL is not required for infection-induced upregulation of DR5 on epithelial and endothelial cells but that it is responsible for infection-induced epithelial cell apoptosis. We observed increased number of apoptotic CD $31^{+}$cells in infected WT mice, so it is possible that apoptosis of $\mathrm{CD} 31^{+}$endothelial cells may contribute to alveolar enlargement. However, there was not a significant decrease in infected $\operatorname{Tnfs} f 10^{-1-}$ mice, so we cannot definitively conclude that TRAIL is inducing apoptosis of endothelial cells. Infection also increased the amount of TRAIL and the number of TRAIL-expressing cells (namely epithelial cells, alveolar macrophages, and inflammatory monocytes), which may bind to DR5 ${ }^{+}$epithelial cells to induce apoptosis. Importantly, administration of rTRAIL or agonistic anti-DR5 antibody to naïve neonatal WT mice (day 0-18) in the absence of infection did not induce alveolar enlargement in later life (day 61) demonstrating that infection-induced upregulation of DR5 expression is required for TRAIL-induced apoptosis. Collectively, these data suggest that concomitant infection-induced increases in both TRAIL and DR5 promote TRAIL-induced apoptosis of lung epithelial cells during early life. This may impair normal alveolar development and lead to emphysemalike alveolar enlargement in later life.

Our observations may be broadly applicable to other pathogenic respiratory infections in early life. RSV strongly 
upregulates TRAIL and its functional receptors DR4 and DR5 in normal human primary bronchial epithelial and small airway epithelial cells ${ }^{27}$ and in primary bronchial epithelial cells from children. ${ }^{34}$ Increased levels of soluble TRAIL were found in the bronchoalveolar lavage fluid of children with severe RSV infection, ${ }^{34}$ and up to $11 \%$ of children with RSV infection develop infantile lobar emphysema. ${ }^{35,36}$ However, a link between alveolar enlargement and TRAIL in RSV has not been made. Influenza $(\mathrm{H} 1 \mathrm{~N} 1, \mathrm{~A} / \mathrm{PR} / 8 / 34)$ infection of mice upregulates TRAIL expression on natural killer cells, CD8 ${ }^{+} \mathrm{T}$ cells and inflammatory monocytes and DR5 on infected T1 $\alpha^{+}$ alveolar epithelial cells. ${ }^{16,17,26}$ Importantly, influenza infections in early life also promote emphysema-like alveolar enlargement that persists in adulthood, ${ }^{4}$ although the role of TRAIL in this process has not been investigated. Therefore, it is unlikely that our observations are restricted only to Chlamydia respiratory infections.

We have previously shown that neonatal and infant, but not adult, Chlamydia respiratory infection promotes the development of persistent AHR (increased transpulmonary resistance and decreased dynamic compliance in response to methacholine) in the absence of antigen sensitization or challenge, , $^{3,12,37}$ which has been confirmed by others. ${ }^{6}$ Here we also demonstrate the mechanisms involved in this observation. We have made the discovery that in the absence of TRAIL, infectioninduced AHR was completely ablated. We also demonstrate that administration of rTRAIL or agonistic anti-DR5 antibody to the lungs of naïve neonatal $\mathrm{BALB} / \mathrm{c}$ mice (from day 0-18) had no effect on the development of persistent AHR (day 61). These data suggest that infection-induced persistent AHR is dependent on TRAIL. We have previously shown that TRAIL and TRAIL responses are pivotal in driving AHR in adult models of AAD ${ }^{15,29}$ TRAIL deficiency and silencing of TRAIL with specific siRNA abolished ovalbumin-induced AHR during AAD.${ }^{15}$ Moreover, delivery of rTRAIL into the airways of naïve adult WT mice induced acute AHR within $24 \mathrm{~h}$ of administration. ${ }^{15}$ This is in contrast to our observation, which can be explained by the fact that rTRAIL administration in our model ceased 6 weeks prior to assessment of AHR. In our current study, we demonstrate that during infection, TRAIL signals through DcR2 to induce persistent AHR. The expression of TRAIL was increased by infection, although that of DcR2 was not. Nevertheless, the increase in TRAIL may drive increased DcR2 signaling. In support of this, blockade of DcR2, but not DcR1, during infection prevented the infection-induced increase in transpulmonary resistance in later life. DcR2 is known to activate NF- $\kappa \mathrm{B},{ }^{21}$ which has been implicated in pulmonary inflammation and AHR. ${ }^{38}$ Using a transgenic mouse model in which activation of NF- $\kappa B$ occurred only in the airway epithelium, Pantano et al., ${ }^{38}$ demonstrated that activation of NF- $\kappa B$ was sufficient to induce AHR in the absence of antigen sensitization and challenge. We demonstrate that blockade of DcR2 during infection significantly decreased NF- $\kappa B$ (p52) activity in the lungs. We have also recently shown that TRAIL induces the expression of the E3 ubiquitin ligase midline-1 (MID1) in the airway wall and that siRNA-induced knockdown of MID1 reduces NF- $\kappa \mathrm{B}$ activity in a house dust mite model of AAD and in an RV infection model. ${ }^{29}$ Thus, we propose that infection-induced TRAIL signals through DcR2 and potentially MID1 and NF- $\mathrm{\kappa B}$-mediated pathways in airway epithelial cells to promote infection-induced persistent AHR. Importantly, this is the first in vivo study to use anti-DcR1 and anti-DcR2-blocking antibodies.

Finally, and most importantly, neutralization of TRAIL from 8 d.p.i. reduced Chlamydia infection-induced histopathology and mucus hypersecretion, and prevented the development of alveolar enlargement and persistent AHR; however, Chlamydia load was unaffected. These data suggest that infection-induced pathology is independent of bacterial load, but dependent on infection-induced TRAIL responses. Thus, treatment of neonates presenting with Chlamydia or other respiratory infections with antibiotics or antivirals may be of limited efficacy, as the induction of TRAIL-mediated responses occur concurrently with the development of infection-related symptoms. However, early intervention of 'at risk' neonates with antibiotics and antivirals may be beneficial. Others have shown that administration of erythromycin every $12 \mathrm{~h}$ for 3 consecutive days from 2 d.p.i., protected against neonatal Chlamydia respiratory infection-induced alveolar enlargement, but had minimal effects on AHR. ${ }^{6}$ Nevertheless, clinical signs (i.e., reduced weight gain) do not become apparent until during infection and more generalized approaches may have broader applicability. Thus, targeting of TRAIL, its receptors, and signaling pathways, may be of widespread therapeutic relevance to early-life respiratory infections and in preventing chronic lung disease.

In summary, our study reveals for the first time that TRAIL promotes the long-term consequences of neonatal Chlamydia respiratory infection and is pivotal in promoting histopathology, inflammation, mucus hypersecretion, alveolar enlargement, and persistent AHR. Chlamydia respiratory infection sensitizes lung epithelial cells to TRAIL-induced apoptosis, which facilitates the development of emphysema-like changes in the lung. Furthermore, TRAIL promotes AHR via DcR2dependent signaling pathways. Collectively, our data suggest that therapeutic targeting of infection-induced TRAIL and TRAIL receptors may be beneficial in reducing the severity of early-life respiratory infection-induced pathology and in preventing the development of associated chronic lung disease.

\section{METHODS}

Ethics statement. This study was performed in strict accordance with the recommendations in the Australian code of practice for the care and use of animals for scientific purposes issued by the National Health and Medical Research Council of Australia. All protocols were approved by the Animal Ethics Committee of The University of Newcastle, Australia.

Neonatal C. muridarum respiratory infection. Neonatal ( $<24 \mathrm{~h}$ old) WT or Tnfsf $10^{-1-}$ BALB/c mice were infected i.n. with the natural mouse pathogen C. muridarum (400 inclusion-forming units, ATCC VR-123, in $5 \mu \mathrm{l}$ sucrose phosphate glutamate buffer. ${ }^{3,12,39}$ Shaminoculated controls received an equivalent volume of sucrose 
Table 1 Histopathological scoring system for mouse lungs

Score 1: Airways inflammation

score (/4)

0

1

2

3

4

Score 2: Vascular inflammation score (/4)

0

1

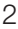

3

4

Score 3: Parenchymal inflammation (at $10 \times$ magnification) score (/5)

0

2

3

4

5
Lack of inflammatory cells around airways-absent

Some airways have small numbers of inflammatory cells-mild

Some airways have significant inflammation-moderate

Majority of airways have some inflammation-marked

Majority of airways have significant inflammation-severe
Lack of inflammatory cells around blood vessels-absent

Some blood vessels have small numbers of inflammatory cells-mild

Some blood vessels have significant inflammation-moderate

Majority of blood vessels have some inflammation-marked

Majority of blood vessels have significant inflammation-severe
Table 2 Characterization of cell subsets

\begin{tabular}{|c|c|}
\hline Cell type & Cell surface antigens \\
\hline Epithelial cells & $\mathrm{T} 1 \alpha^{+} \mathrm{CD} 31^{-}$ \\
\hline Endothelial cells & $\mathrm{T} 1 \alpha^{-} \mathrm{CD} 31^{+}$ \\
\hline Alveolar macrophage & $\mathrm{F} 4 / 80^{+} \mathrm{CD} 11 \mathrm{~b}^{-} \mathrm{CD} 11 \mathrm{c}^{+}$ \\
\hline Inflammatory monocyte & $\mathrm{F} 4 / 80^{+} \mathrm{CD} 11 \mathrm{~b}^{+} \mathrm{Gr}-1^{+}$ \\
\hline Neutrophil & $\mathrm{F} 4 / 80^{-} \mathrm{CD} 11 \mathrm{c}^{-} \mathrm{CD} 11 \mathrm{~b}^{\mathrm{hi}} \mathrm{Gr}-1^{\mathrm{hi}}$ \\
\hline $\mathrm{mDC}$ & $\mathrm{F}_{4 / 80^{-}} \mathrm{CD} 11 \mathrm{c}^{+} \mathrm{CD} 11 \mathrm{~b}^{+} \mathrm{PDCA}^{-}$ \\
\hline pDC & 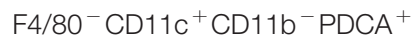 \\
\hline $\mathrm{CD}^{+}{ }^{+} \mathrm{T}$-cell & $\mathrm{CD}^{+}{ }^{+} \mathrm{CD} 4^{+} \mathrm{CD}^{-}$ \\
\hline $\mathrm{CD}^{+}{ }^{+} \mathrm{T}-$ cell & $\mathrm{CD}^{+} \mathrm{CD}^{-} \mathrm{CD}^{+}$ \\
\hline
\end{tabular}

Biosciences, North Ryde, New South Wales, Australia) for 15 min to prevent non-specific immunofluorescent staining. In optimization experiments, test antibodies were compared with their isotype controls. No significant non-specific staining was identified with isotype controls at the concentration used compared with unstained controls during these experiments. Blocked cells were stained for the surface markers CD3, CD4, CD8, F4/80, CD11c, CD11b, Gr-1, T1 $\alpha$, CD31, TRAIL, and DR5 (Biolegend, Karrinyup, Western Australia, Australia) and PDCA (Miltenyi, North Ryde, New South Wales, Australia). Apoptotic cells were quantified using an FITC annexin-V dead cell apoptosis kit (Invitrogen, Mount Waverely, Victoria, Australia). Cells were fixed with $4 \%$ paraformaldehyde and cell populations analyzed using FACSCanto and FACSDiva software (BD Biosciences). ${ }^{37,40,45,46}$ Inflammatory cells subsets were defined as described in Table 2 and representative gating strategies are shown in Supplementary Figures 2-7.

Enzyme-linked immunosorbent assay. Total protein was isolated from lung homogenates using SurePrep RNA/DNA/Protein Purification kits. Purified total protein was quantified using BCA Protein Assay Kits (PIERCE, Scoresby, Victoria, Australia) according to manufacturer's instructions. The concentration of murine TRAIL in lung homogenates was analyzed by ELISA using paired antibodies (R\&D systems, Gymea, New South Wales, Australia). ${ }^{15} \mathrm{NF}-\kappa B$ subunit (p52, RelB, p65, and p50) activity was determined using the NF- $\kappa B$ family transcription factor assay kit (Active Motif, Carlsbad, CA) according to the manufacturer's instructions. ${ }^{29}$

Quantification of mRNA expression by real-time quantitative PCR. Total RNA was isolated from lung homogenates using SurePrep RNA/ DNA/Protein Purification kits. ${ }^{37}$ Extracted RNA was treated with DNAseI (Sigma-Aldrich, Castle Hill, New South Wales, Australia) and reverse-transcribed using Bioscript (Bioline, Alexandria, New South Wales, Australia) and random hexamer primers (Invitrogen). Relative abundance of cytokine cDNA was determined by comparison with the reference gene hypoxanthine-guanine phosphoribosyltransferase by real-time PCR using an Eppendorf RealPlex 2 System (Eppendorf, North Ryde, New South Wales, Australia). Custom-designed primers (IDT, Coralville, IA) were used for the analysis of mRNA expression for TRAIL (For; 5'-CCCTGCTTGCAGGTTAAGAG-3' Rev; $5^{\prime}$-GGCCTAAGGTCTTTCCATCC-3'), DR5 (For; 5' -AACGGC TTGGGCATCTTGGCA-3' Rev; 5'-TGCACAGAGTTCGCACT TTCGGG-3'), DcR1 (For; 5'-TTTCCGGAATCATGCCGCCCA-3' Rev; $5^{\prime}$-AGGAACAGCCAGTTTCTGGGATTTG-3'), and DcR2 (For; 5'-TGTCCCGCTGGTGAATACTGGT-3' Rev; 5'-GGATTCGCAG GGCGCCTTG- $\left.3^{\prime}\right)$.

Lung function. Lung function, in terms of AHR, was measured in anesthetized mice using invasive whole-body plethysmography (Buxco, Wilmington, NC) by determination of transpulmonary 
resistance and dynamic compliance in response to increasing doses of nebulized methacholine (Sigma) at 61 d.p.i. ${ }^{47-49}$

Alveolar enlargement. Average alveolar diameter was evaluated at 61 d.p.i. by determination of the mean linear intercept. ${ }^{3,50}$

In vivo administration of rTRAIL, agonistic anti-DR5, and antiDcR1, and anti-DcR2-blocking antibodies. WT mice were treated with $0.5 \mu \mathrm{gg}^{-1}$ body weight of murine rTRAIL (R\&D systems), agonistic anti-DR5 antibody (MD5-1), and neutralizing anti-DcR1 antibody (mDcR1-3), or anti-DcR2 antibody (mDcR2-1) i.n. 0, 3, 6, 9, 12, 15, and 18 d.p.i. ${ }^{15}$ Sham-treated controls received sterile PBS or isotype control i.n.

In vivo neutralization of TRAIL. WT mice were treated with neutralizing anti-TRAIL antibody (N2B2) or isotype control $\left(12.5 \mu \mathrm{gg}^{-1}\right.$ body weight i.p.) every second day from day $8-18 .^{51,52}$

Statistics. Results are represented as \pm s.e.m., which is representative of 2-3 independent experiments with each experimental iteration consisting of at least three mice. Statistical significance for multiple comparisons was determined by one-way analysis of variance with Bonferroni post test, or non-parametric equivalent, where appropriate using GraphPad Prism Software version 5 (San Diego, CA).

SUPPLEMENTARY MATERIAL is linked to the online version of the paper at http://www.nature.com/mi

\section{ACKNOWLEDGEMENTS}

This work was supported by grants from the National Health and Medical Research Council of Australia and the Australian Research Council.

\section{DISCLOSURE}

The authors declared no conflict of interest.

c) 2014 Society for Mucosal Immunology

\section{REFERENCES}

1. Hansbro, P.M., Beagley, K.W., Horvat, J.C. \& Gibson, P.G. Role of atypical bacterial infection of the lung in predisposition/protection of asthma. Pharmacol. Ther. 101, 193-210 (2004).

2. Hansbro, N.G., Horvat, J.C., Wark, P.A. \& Hansbro, P.M. Understanding the mechanisms of viral induced asthma: new therapeutic directions. Pharmacol. Ther. 117, 313-353 (2008).

3. Horvat, J.C. et al. Early-life chlamydial lung infection enhances allergic airways disease through age-dependent differences in immunopathology. J. Allergy Clin. Immunol. 125, 617-625. 625 e611-625 e616 (2010).

4. You, D. et al. Inchoate CD8 + T cell responses in neonatal mice permit influenza-induced persistent pulmonary dysfunction. J. Immunol. 181 3486-3494 (2008).

5. Harrison, H.R., Taussig, L.M. \& Fulginiti, V.A. Chlamydia trachomatis and chronic respiratory disease in childhood. Pediatr. Infect. Dis. 1, 29-33 (1982).

6. Jupelli, M. et al. Neonatal chlamydial pneumonia induces altered respiratory structure and function lasting into adult life. Lab Invest. 91 1530-1539 (2011).

7. Weiss, S.G., Newcomb, R.W. \& Beem, M.O. Pulmonary assessment of children after chlamydial pneumonia of infancy. J. Pediatr. 108, 659-664 (1986).

8. Schachter, J. et al. Prospective study of perinatal transmission of Chlamydia trachomatis. JAMA 255, 3374-3377 (1986).

9. Zaitsu, M. Does Chlamydia pneumoniae infection trigger to development of asthma in wheezy infants? J. Asthma 46, 967-968 (2009).

10. Webley, W.C. et al. The bronchial lavage of pediatric patients with asthma contains infectious. Chlamydia. Am. J. Respir. Crit. Care Med. 171 1083-1088 (2005).

11. Webley, W.C. et al. Occurrence of Chlamydia trachomatis and Chlamydia pneumoniae in paediatric respiratory infections. Eur. Respir. J. 33, 360-367 (2009).
12. Horvat, J.C. et al. Neonatal chlamydial infection induces mixed T-cell responses that drive allergic airway disease. Am. J. Respir. Crit. Care Med. 176, 556-564 (2007).

13. Collison, A, Foster, P.S. \& Mattes, J. Emerging role of tumour necrosis factorrelated apoptosis-inducing ligand (TRAIL) as a key regulator of inflammatory responses. Clin. Exp. Pharmacol. Physiol. 36, 1049-1053 (2009).

14. Wiley, S.R. et al. Identification and characterization of a new member of the TNF family that induces apoptosis. Immunity 3, 673-682 (1995).

15. Weckmann, M. et al. Critical link between TRAlL and CCL20 for the activation of $\mathrm{TH} 2$ cells and the expression of allergic airway disease. Nat. Med. 13, 1308-1315 (2007)

16. Brincks, E.L., Katewa, A, Kucaba, T.A., Griffith, T.S. \& Legge, K.L. CD8 T cells utilize TRAIL to control influenza virus infection. J. Immunol. 181, 4918-4925 (2008).

17. Herold, S. et al. Lung epithelial apoptosis in influenza virus pneumonia: the role of macrophage-expressed TNF-related apoptosis-inducing ligand. J. Exp. Med 205, 3065-3077 (2008).

18. Kayagaki, N. et al. Type I interferons (IFNs) regulate tumor necrosis factorrelated apoptosis-inducing ligand (TRAIL) expression on human T cells: a novel mechanism for the antitumor effects of type I IFNs. J. Exp. Med. 189, 1451-1460 (1999)

19. Koga, Y, Matsuzaki, A, Suminoe, A, Hattori, H\& Hara, T. Neutrophil-derived TNF-related apoptosis-inducing ligand (TRAIL): a novel mechanism of antitumor effect by neutrophils. Cancer Res. 64, 1037-1043 (2004).

20. Stary, G. et al. Plasmacytoid dendritic cells express TRAIL and induce CD4 + T-cell apoptosis in HIV-1 viremic patients. Blood 114, 3854-3863 (2009).

21. Degli-Esposti, M.A. et al. The novel receptor TRAIL-R4 induces NFkappaB and protects against TRAlL-mediated apoptosis, yet retains an incomplete death domain. Immunity 7, 813-820 (1997).

22. Pan, G. et al. An antagonist decoy receptor and a death domain-containing receptor for TRAIL. Science 277, 815-818 (1997).

23. Pan, G. et al. The receptor for the cytotoxic ligand TRAlL. Science 276, 111-113 (1997).

24. Sheridan, J.P. et al. Control of TRAlL-induced apoptosis by a family of signaling and decoy receptors. Science 277, 818-821 (1997).

25. Schneider, P. et al. TRAIL receptors 1 (DR4) and 2 (DR5) signal FADDdependent apoptosis and activate NF-kappaB. Immunity 7, 831-836 (1997).

26. Ishikawa, E, Nakazawa, M, Yoshinari, M \& Minami, M. Role of tumor necrosis factor-related apoptosis-inducing ligand in immune response to influenza virus infection in mice. J. Virol. 79, 7658-7663 (2005).

27. Kotelkin, A, Prikhod'ko, E.A., Cohen, J.I., Collins, P.L. \& Bukreyev, A. Respiratory syncytial virus infection sensitizes cells to apoptosis mediated by tumor necrosis factor-related apoptosis-inducing ligand. J. Virol. 77, 9156-9172 (2003).

28. You, D. et al. Exposure of neonates to respiratory syncytial virus is critical in determining subsequent airway response in adults. Respir. Res. 7, 107 (2006).

29. Collison, A. et al. The E3 ubiquitin ligase midline 1 promotes allergen and rhinovirus-induced asthma by inhibiting protein phosphatase 2A activity. Nat. Med. 19, 232-237 (2013).

30. Steinwede, K. et al. TNF-related apoptosis-inducing ligand (TRAIL) exerts therapeutic efficacy for the treatment if pneumococcal pneumonia in mice. J. Exp. Med. 209, 1937-1952 (2012).

31. Asquith, K.L. et al. Interleukin-13 promotes susceptibility to chlamydial infection of the respiratory and genital tracts. PLoS Pathog. 7, e1001339 (2011)

32. Beagley, K.W., Huston, W.M., Hansbro, P.M. \& Timms, P. Chlamydial infection of immune cells: altered function and implications for disease. Crit. Rev. Immunol. 29, 275-305 (2009).

33. Qiu, H. et al. Type I IFNs enhance susceptibility to Chlamydia muridarum lung infection by enhancing apoptosis of local macrophages. J. Immunol. 181, 2092-2102 (2008).

34. Bem, R.A. et al. Potential role of soluble TRAIL in epithelial injury in children with severe RSV infection. Am. J. Respir. Cell. Mol. Biol. 42, 697-705 (2010).

35. Kern, S, Uhl, M, Berner, R, Schwoerer, T \& Langer, M. Respiratory syncytial virus infection of the lower respiratory tract: radiological findings in 108 children. Eur. Radiol. 11, 2581-2584 (2001)

36. Newman, B \& Yunis, E. Lobar emphysema associated with respiratory syncytial virus pneumonia. Pediatr. Radiol. 25, 646-648 (1995). 
37. Starkey, M.R. et al. Constitutive production of IL-13 promotes early-life Chlamydia respiratory infection and allergic airway disease. Mucosal Immunol 6, 569-579 (2012).

38. Pantano, C. et al. Nuclear factor-kappaB activation in airway epithelium induces inflammation and hyperresponsiveness. Am. J. Respir. Crit. Care Med. 177, 959-969 (2008).

39. Starkey, M.R. et al. Chlamydia muridarum lung infection in infants alters hematopoietic cells to promote allergic airway disease in mice. PLoS One 7, e42588 (2012).

40. Horvat, J.C. et al. Chlamydial respiratory infection during allergen sensitization drives neutrophilic allergic airways disease. J. Immunol. 184, 4159-4169 (2010).

41. Fung, K.Y. et al. Interferon- $\epsilon$ protects the female reproductive tract from viral and bacterial infection. Science 339, 1088-1092 (2013).

42. Preston, J.A. et al. Inhibition of allergic airways disease by immunomodulatory therapy with whole killed Streptococcus pneumoniae. Vaccine 25 , 8154-8162 (2007)

43. Preston, J.A. et al. Streptococcus pneumoniae infection suppresses allergic airways disease by inducing regulatory T-cells. Eur. Respir. J. 37, 53-64 (2011).

44. Thorburn, A.N. et al. Pneumococcal conjugate vaccine-induced regulatory T cells suppress the development of allergic airways disease. Thorax 65 , 1053-1060 (2010).
45. Beckett, E.L. et al. TLR2, but not TLR4, is required for effective host defence against Chlamydia respiratory tract infection in early life. PLoS One 7, e39460 (2012).

46. Essilfie, A.T. et al. Combined Haemophilus influenzae respiratory infection and allergic airways disease drives chronic infection and features of neutrophilic asthma. Thorax 67, 588-599 (2012).

47. Essilfie, A.T. et al. Haemophilus influenzae infection drives IL-17-mediated neutrophilic allergic airways disease. PLoS Pathog. 7, e1002244 (2011).

48. Thorburn, A.N., Foster, P.S., Gibson, P.G. \& Hansbro, P.M. Components of Streptococcus pneumoniae suppress allergic airways disease and NKT cells by inducing regulatory T cells. J. Immunol. 188, 46114620 (2012).

49. Kaiko, G.E. et al. Chlamydia muridarum infection subverts dendritic cell function to promote Th2 immunity and airways hyperreactivity. J. Immunol. 180, 2225-2232 (2008).

50. Robbesom, A.A. et al. Morphological quantification of emphysema in small human lung specimens: comparison of methods and relation with clinical data. Mod. Pathol. 16, 1-7 (2003).

51. Cretney, E. et al. TNF-related apoptosis-inducing ligand (TRAIL)/Apo2L suppresses experimental autoimmune encephalomyelitis in mice. Immunol. Cell Biol. 83, 511-519 (2005).

52. Takeda, $\mathrm{K}$. et al. Induction of tumor-specific T cell immunity by antiDR5 antibody therapy. J. Exp. Med. 199, 437-448 (2004). 